\title{
Sources of evidence: useful databases
}

\author{
Jeyanthi John
}

Senior Researcher, Centre for Evidence-based Dentistry, Oxford, UK

\author{
Previously in this series I have described two of the major biomedical databases, \\ Medline and the Cochrane Library. In this article I briefly outline a few of the other \\ available sources of literature. \\ Evidence-Based Dentistry (2004) 5, 24-27. doi:10.1038/sj.ebd.6400232
} necessary to look outside the standard medical databases such as Medline and the Cochrane Library. Many specialised databases exist that cover topics relating to biomedical sciences, health, education and social care. Some of them include journals not indexed on Medline. These are useful if you are looking for papers in such specialist areas, or would like to make your search more exhaustive, eg, databases charge for access, although many are available to UK National Health Service (NHS) staff with an Athens password (available through the Athens website at www.athens.nhs.uk). Academics in the UK and abroad usually have access to at least one of these sites through their library or online services.

\begin{abstract}
AMED
AMED (Allied and Complementary Medicine Database) (Figure 1) is a unique bibliographic database produced by the Health Care Information Service of the British Library. It covers a selection of journals in three separate subject areas:

- several professions allied to medicine,

- complementary medicine, and

- palliative care.

AMED covers around 596 journals, from 1985, including many not indexed by other biomedical sources. The scope of coverage is mainly European with the majority of titles published in English. Each record includes controlled indexing terms using the AMED Thesaurus based on MeSH (Medline indexing terms). In addition to the specialist journals on
\end{abstract}

In some areas of research it may be for a systematic review. Several of these
AMED's subjects, a range of key general journals are checked for relevant articles.

\section{ASSIA}

ASSIA (Applied Social Sciences Index and Abstracts on the Web) is an indexing and abstracting tool dealing with health, social services, psychology, sociology, economics, politics, race relations and education. Updated monthly, ASSIA provides a comprehensive source of social science and health information for the practical and academic professional. It currently contains over 312000 records from 650 journals in 16 different countries, including the UK and US.

\section{CancerLit}

Produced by the US National Cancer Institute, CancerLit is an important source of bibliographic information pertaining to all aspects of cancer therapy, including experimental and clinical cancer therapy; chemical, viral and other cancer-causing agents; mechanisms of carcinogenesis; biochemistry, immunology and physiology of cancer; and mutagen and growth factor studies. Some of the information in CancerLit is derived from the Medline database. Approximately 200 core journals contribute a large proportion of the $750000+$ records in this database, dating from 1983 onwards. In addition, other information is drawn from proceedings

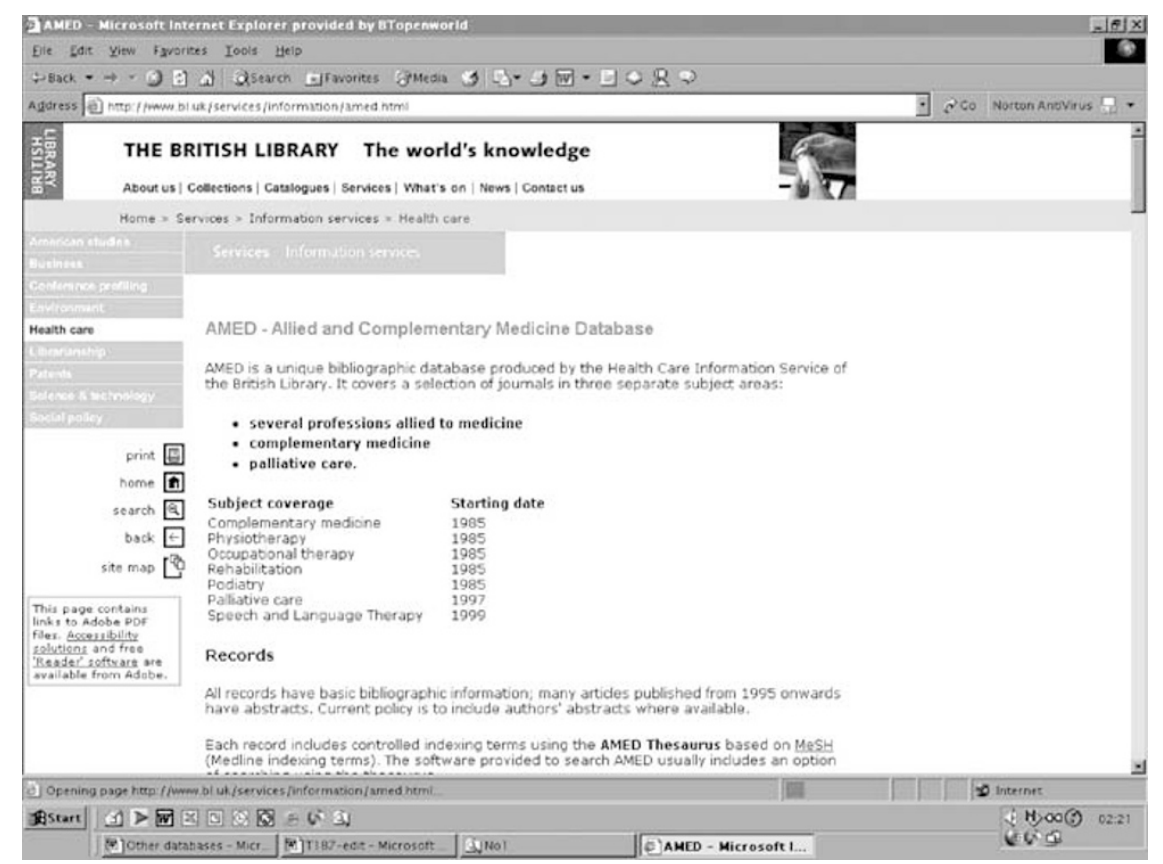

Figure 1. AMED web page. 
of meetings, government reports, symposia reports, theses and selected monographs.

\section{CINAHL}

The CINAHL (Cumulative Index of Nursing and Allied Health Literature) database contains more than 200000 citations from 1983 to the present, with abstracts to articles published in more than 650 English-language nursing and allied health journals. It also covers literature from health education, education, health sciences and consumer health.

CINAHL is searched by entering key words and phrases and using Boolean operators, such as "and" and "or", just as in Medline. In fact, you can search CINAHL and Medline databases simultaneously by selecting both databases when you begin your search. There is a thesaurus and options for limiting the search, such as by date. At the end of the search, you can select the citations and store them. When you have all the citations you want, you can choose to either to print the results, download them into a file or e-mail them.

\section{Dissertation abstracts}

Dissertation Abstracts Online (DAIC) is a definitive subject, title and author guide to virtually every American dissertation accepted at an accredited institution since 1861. Selected Masters theses have been included since 1962. Since 1988, the database includes citations for dissertations from 50 British universities that have been collected by and microfilmed at the British Document Supply Centre. Beginning with DAIC Volume 49, Number 2 (Spring 1988), citations and abstracts from Section C, Worldwide Dissertations (formerly European Dissertations), have been included in the file.

\section{EMbase}

EMbase (Excerpta Medica database) is a bibliographic database of biomedical and pharmaceutical literature, produced by Elsevier Science. It contains more than 9 million records from over 4000 journals encompassing 1974 to the present. Patents, handbook monographs and dissertations are not included. EMbase can be accessed by anyone who is an NHS employee through the Athens website, as above.

EMbase contains a thesaurus called EMTREE with indexing that is consistent

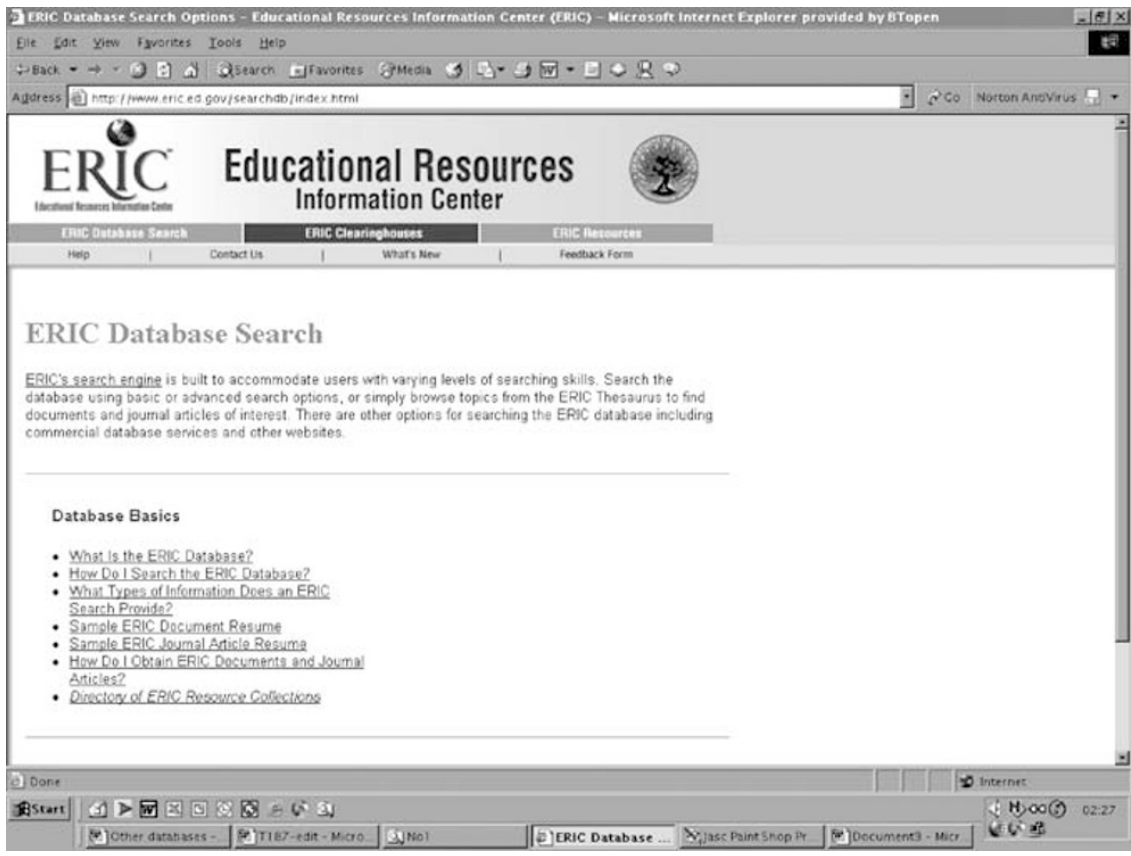

Figure 2. Educational resources information webpage.

across EMbase and Medline records. You can use Boolean operators and limit your searches using options available on the screen.

\section{ERIC}

ERIC (Educational Resources Information Centre; www. eric.ed.gov/) (Figure 2) is a database of education literature. Sponsored by the US Department of Education, ERIC consists of two files: Resources in Education, covering the document literature, and Current Index to Journals in Education, covering published journal literature from over 775 periodicals. The database also includes the full text of ERIC Digest records. These 1- to 2-page records, written specifically for teachers, administrators and other practitioners, provide an overview of information on a given topic and supply references with more detailed information. In January 2004, the Department of Education will begin to implement a re-engineering plan for ERIC. However, the ERIC database will continue to grow, as thousands of documents selected by the ERIC clearinghouses throughout 2003 will be added. Towards the end of 2004 a new model for the database will appear.

\section{PsycINFo}

PsycINFO is an electronic bibliographic database produced by the American Psychological Association (Figure 3).
It provides abstracts and citations to literature in the behavioural sciences and mental health and covers journals in many health-related fields including psychiatry, epidemiology, health administration, rehabilitation and pharmacology.

This collection covers more than 1.9 million records from over 1800 titles, with comprehensive coverage of more than 650 titles. Articles of psychological relevance are selected by PsycINFO staff from another 300 additional journals. For example, articles about tobacco use are included if there is a behavioural component but not if they focus solely on the physical effects. Journals are included from 1887 to the present and books from 1987-date. Books, dissertations and reports are only included if they are in English, but journal articles are from more than 24 languages.

PsycINFO can also be searched in the same way as CINAHL and Medline, using key terms or phrases. Icons appear at the top of the screen to help you with searching so you can, for example, combine or limit searches, or search for a particular author or search in a particular journal. Options to limit searches, eg, by date, appear at the bottom of the search screen. Search terms are automatically mapped to subject headings so uncheck the box if you do not want to do this. At the end of the search you can 


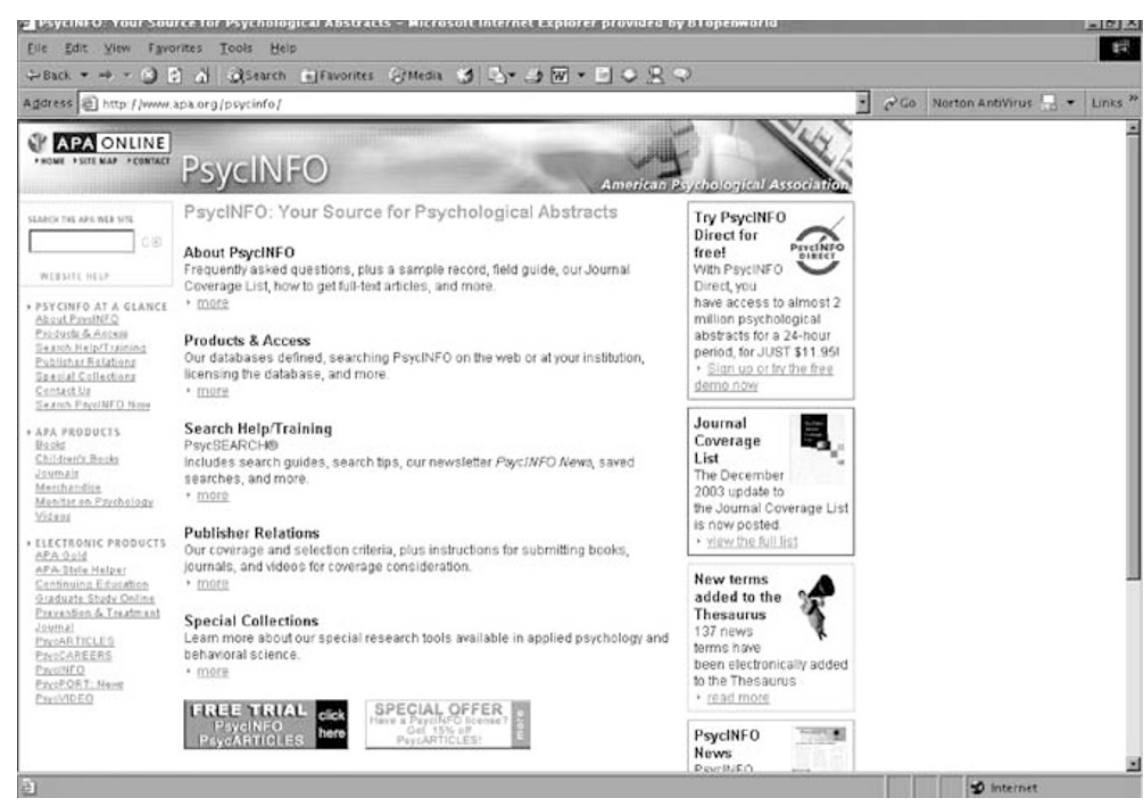

Figure 3. PsycINFO webpage.

choose to either print, e-mail or save selected citations.

\section{HTA Database}

Health Technology Assessment (HTA) database (Figure 4) studies the medical, social, ethical and economic implications of development, diffusion and use of health technology (eg, vaccines, devices and procedures). These studies help inform policy decisions and improve the quality and cost-effectiveness of healthcare. The HTA database contains information on healthcare technology assessments and is produced in collaboration with the International Network of Agencies for Health Technology Assessment (INAHTA). It can be assessed on the website of the NHS Centre for Reviews and Dissemination (CRD), nhscrd.york.ac.uk. The HTA database is also included in the Cochrane Library.

The database was created in 1998 but includes publications from 1988. It does not operate quality inclusion criteria to select records but all records are of publications and projects from nationally funded health technology assessment organisations. Most of the records contain a web-link to the original document, or to a summary of the document, where this is electronically available. Full contact details for each agency are provided for each record so you can request a paper copy if required or if you would like more information about a project. The CRD information service will undertake searches for you if you do not have access to their databases. Their contact details are as follows: telephone + 44 (0)1904 433707 (9am$5.15 \mathrm{pm}$, Monday-Friday), fax +44 (0)1904 433661, e-mail revdis@york. ac.uk.

\section{LILACS}

LILACS (Latin American and Caribbean Health Science Literature) is the collection of databases for recording and disseminating the scientific work of Latin database access page.
American and Caribbean Health professionals. It is the result of a co-operative effort between the constituent countries. LILACS contains information from more than 670 journals from 1982 onwards with abstracts in English, Portuguese or Spanish. Of these 670 journals indexed, only 41 overlap with Medline/EMbase.

LILACS is edited by BIREME (Latin American and Caribbean Health Science Information Center; www.bireme.br), in the city of São Paulo, Brazil. Currently, BIREME is an agency of the Pan-American Health Organization/World Health Organization.

\section{Oxford Pain database}

This database is dedicated to information on the management of pain. It can be accessed at www.jr2.ox.ac.uk/bandolier/ booth/painpag/index.html. It collates systematic reviews with pain as an outcome. The site is freely available to anyone who is interested in pain management either in a professional or personal capacity. There are several topics, including general information about pain, a league table of analgesics, and information about different types of acute pain and chronic pain.

This is not an exhaustive database of pain research, but it does provide a useful summary of each subject. All the information is based on the best evidence available and each topic starts off with a clinical bottom line.

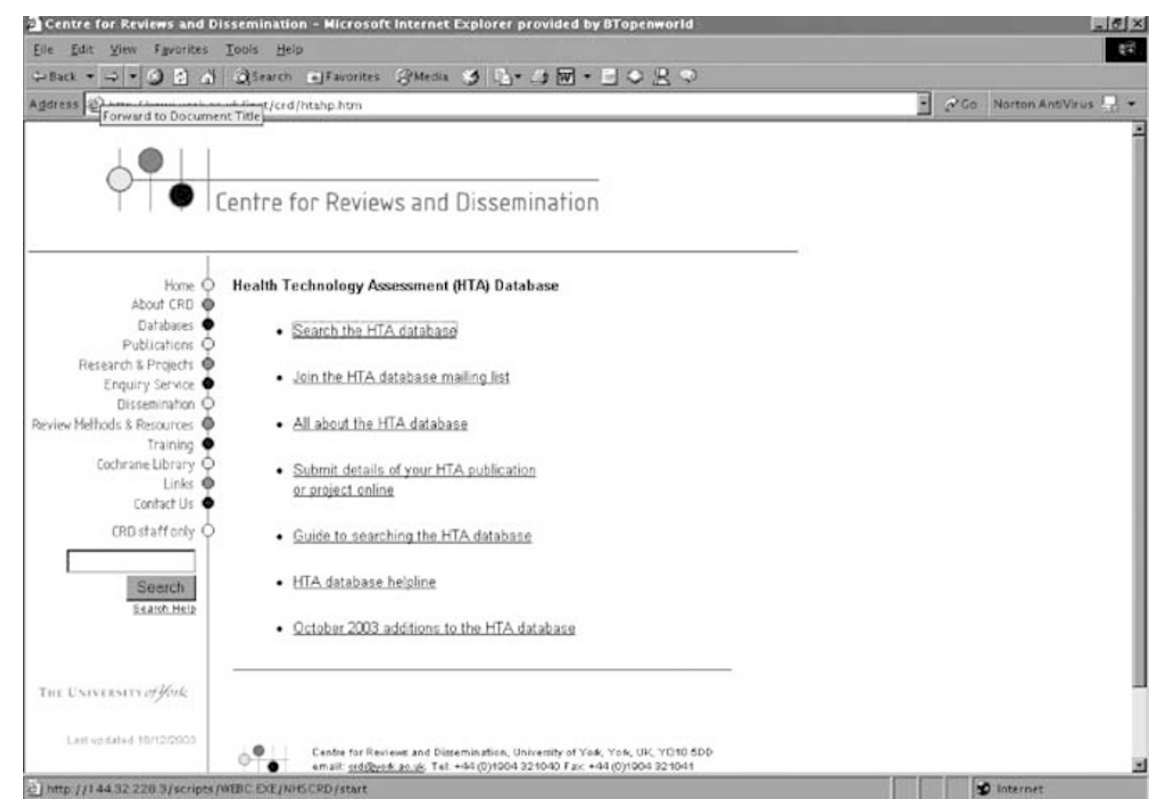

Figure 4. Centre for Reviews and Dissemination, York-health technology assessment 


\section{Web of Science}

The Web of Science provides access to:

- The Science Citation Index and Scisearch-the gateway to current and retrospective bibliographic information, author abstracts and cited references found in 3700 of the world's leading scholarly science and technical journals in more than 100 disciplines. The Science Citation Index Expanded Format and SciSearch cover more than 5800 journals. Available 1995-present as Web of Science, and with local CD-ROM access only for 1986-1993. There is also a paper index for 1945 to date.

- The Social Science Citation index and Social SciSearch-provide access to current and retrospective bibliographic information, author abstracts and cited references found in over 1700 of the world's leading scholarly social sciences journals, encompassing more than 50 disciplines. They also cover individually selected, relevant items from approximately 3300 leading, international science and technology journals. Again, these are available 1995-present as Web of Science, with local CD-ROM access only for 1986-1992.

The wide range of databases noted above are some of those most often cited in data sources of systematic reviews published in Evidence-Based Dentistry. However, this is far from an exhaustive list. Some may recall that for the sys- tematic review of water fluoridation, ${ }^{1} 25$ different databases were searched, many of which I have not included above.

The average practitioner may have little need to visit these sources regularly, if at all, but it is important to be aware of those mentioned here if engaged in research, or when conducting systematic reviews in particular, along with using Medline, the Cochrane library and the more specialised databases.

1. McDonagh MS, Whiting PF, Wilson PM, et al Systematic review of water fluoridation. $\mathrm{Br}$ Med J 2000; 321:855-859. 